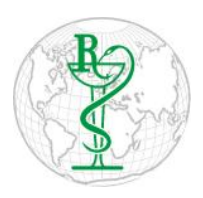

INDO GLOBAL JOURNAL OF

PHARMACEUTICAL SCIENCES

ISSN 2249- 1023

\title{
From Conventional Treatment to Targeted Antimalarial Therapy: Building Up on Innovations
}

\author{
Saini Akash ${ }^{1 *}$, Gupta Pritee ${ }^{2}$ \\ ${ }^{1}$ K.R. Mangalam University, Gurgaon, Haryana, India \\ ${ }^{2}$ College of Pharmaceutical Sciences, Raj Kumar Goel Institute of Technology, Ghaziabad, Uttar Pradesh, India
}

Address for Correspondence: Saini Akash, akashsaini.saini@gmail.com ; gupta.pg.priti@gmail.com

Received:
28.03.2019
Accepted:
29.03.2020
Published:
30.03.2021
Keywords
Malaria, Drug
resistance,
Challenges,
Nanotechnology.

\begin{abstract}
Despite many technological advancements and innovations, infectious disease like malaria continues to be one of the greatest health challenges worldwide. The conventional drug therapy has various limitations. There are various challenges faced by drug therapy and the eradication agenda. However, various new strategies are being investigated not only to treat the parasitic disease but to prevent its spread and relapse. Nanotechnology-based approaches (diagnosis treatment and prevention), reconsidering drugs with limitations, repurposing of drugs, and development of new drugs by modifying existing compounds, reoptimizing available antimalarial, creating new combinations, etc. are some of them. The use of herbal drugs for antimalarial therapy has provided a novel approach having lesser side effects, safety, and more efficacies. Recent developments like investigating new targets for antimalarial drugs, newer and more effective mechanisms for attacking the parasite, targeting organ system and multiple stages of the parasitic life cycle and many other newer approaches may revolutionize the way we are fighting the malaria parasite soon. () 2020 iGlobal Research and Publishing Foundation. All rights reserved.
\end{abstract}

Cite this article as: Saini, A.; Gupta, P. From conventional treatment to targeted antimalarial therapy: building up on innovations. Indo Global J. Pharm. Sci., 2021; 11(1): 15-27. DOI: http://doi.org/10.35652/IGJPS.2021.111003.

\section{INTRODUCTION}

Malaria is the most prevalent parasitic disease of human beings. It is transmitted in 109 countries out of which 45 are in Africa [1]. Five species of the apicomplex protozoan of genus Plasmodium transmitted to humans by the bites of the female mosquito vector of the Anopheles genus are responsible for malarial infections in human beings. Most of the cases are due to Plasmodium falciparum or $\mathrm{P}$. vivax, but infections in humans can also be caused by P. ovale, P. malariae, and in some parts of South East Asia by the monkey malaria P. knowlesi. Various strains of Plasmodium resistant to many of available drugs have appeared, making the efficient therapy of malaria more demanding [2]. The dosage regimens for Malaria therapy are complex and are known to reduce patient adherence to the therapy. Lack of patient compliance has led to the emergence of multidrug-resistant strains of parasites [3]. The complexity of the chemotherapy is mainly attributed to the different stages of the parasite life cycle. It can be treated with antimalarial combinations; however, toxicity, side- effects, low drug solubility, and drug resistance continue to drive drug-delivery research. Nanoparticles, due to their size scale, have unusual physicochemical properties that differ substantially from those of the bulk materials of the same composition [4]. Nanoparticulate drug delivery systems represent a promising approach for obtaining desirable druglike properties by altering the biopharmaceutics and pharmacokinetics property of the drug molecule.

\section{EPIDEMIOLOGY}

The main factors determining the transmission intensity of malaria parasite are the density, biting habits, longevity and efficiency of the vector mosquito as follows:

Of the known more than 400 anopheline species, only around 25 are good vectors [5].

Effective ones are the ones with longevity, robustness to the environmental changes, and occurrence in highly dense 


\section{Indo Global Journal of Pharmaceutical Sciences, 2021; 11(1): 15-27}

tropical climates, with rapid breeding patterns and with specific human-biting preferences [6].

Childhood period (early) is more prone to mortality and morbidity and by adulthood infections are asymptomatic.

Infections may have annual as well as seasonal patterns like; increment in rainy season [7].

The disease does not provide protective immunity and asymptomatic disease can occur at any age [8].

\section{PATHOGENESIS}

Infected Red Blood Corpuscles adheres to walls of vessels and each other (platelet mediated agglutination) or non-infected RBCs (resetting). The attachment causes sequestration of infected erythrocytes into vital organs like brain (particularly), where the parasites thus collected interferes with the functioning of endothelium, metabolism and microcirculatory flow. P vivax, $\mathrm{P}$ ovale, and $\mathrm{P}$ malariae invade red blood cells selectively. The infected red blood cells become enlarged and gets deformed. By contrast, as P falciparum matures, the infected red blood cell becomes more spherical and rigid. In severe falciparum malaria, non-infected erythrocytes also become less deformable, which compromises flow through the partially obstructed capillaries and venules and shortens their survival. Rupture of Schizont releases parasite and host cellular material into the blood, which activates monocytes and macrophages and induces the release of pro-inflammatory cytokines, causing fever and other pathological effects $[9,10$, $11,12]$.

\section{DRUG TRANSPORT INTO THE PARASITE}

The main aim of drug therapy is to provide high drug concentration in the intercellular parasitophorous vacuole hosting the plasmodium [13, 14]. There are multiple membranes to be crossed by the antimalarial drugs to access the intra-parasitic targets. These are in orderly fashion starting from:

The cell membrane of the host

The vacuolar membrane containing the parasite

The cell membrane of the parasite

Organelle membrane (depends upon site of the action)- food vacuole/endoplasmic reticulum [15].

$\mathrm{pH}$ differs in these compartments in infected erythrocytes which might be exploited for selective delivery using $\mathrm{pH}$ sensitive nanosystems [16].

\section{LIMITATIONS OF CONVENTIONAL THERAPY}

There are frequent failures associated with chemotherapy due to various factors:

1. Intrinsic factors associated with the disease:

a. Transmission conditions

b. Difficulty in controlling the spread in the tropical areas

c. Parasitic life cycle

d. Resistance to drug therapy

e. Immunocompromised patients suffering from malaria and HIV co-infection

f. Complexity related to dosage regimens recommended

2. Extrinsic factors related to drug usage:

a. The quality of distributed drugs in some countries is poor

b. Drug interactions

c. Fewer toxic drugs are generally not available

d. Insecticidal resistance in the vector

e. Social conditions of affected populations

f. Market Failure related to the inability of most consumers to pay for their drug therapy thus making the market for manufacturing antimalarial drugs non-profitable [17].

\section{DRUG RESISTANCE}

The already limited antimalarial armory is now severely compromised due to the remarkable ability of the parasite to develop resistance to these compounds. However, the distribution of such type of resistance to anyone of particular drug greatly varies geographically; there are areas with a high prevalence of complete drug resistance, while in other areas there is a spectrum of sensitivity to various drugs [18].

The resistance might have genetic basis related to the selection of parasites harboring polymorphisms, particularly point mutations, associated with reduced drug sensitivity, is the primary basis for drug resistance in malaria parasites $[19,20]$ and the ones exposed to sub-therapeutic drug concentrations are preferentially selected.

Other reasons might be unregulated use, inadequate drug regimens or use of long half-life drugs singly or with Artemisinin combination therapies [21, 22]. Recrudescence, the reappearance of an infection after a period of quiescence have also been seen in case of a Artemisinin treatment specially in $\mathrm{P}$. falciparum in some areas, however, the mechanism for such a happening remains unclear for the time being perhaps due to the complexity of mechanisms of action and potential targets of Artemisinin and the research related to parasite dormancy might just provide the mechanism in the near future remains to be seen[23, 24]. 


\section{Indo Global Journal of Pharmaceutical Sciences, 2021; 11(1): 15-27}

\section{CHALLENGES}

Challenges faced by anti-malarial therapy:

a. There is an urgent need to develop new strategies especially while considering the increased incidences of drug resistance and that too in the endemic areas of P. falciparum and combined therapy might be the answer.

b. Toxicity levels of drugs vary considerably, thereby limiting their use due to lack of specificity to target cells in most of the formulations. Secondly, therapeutic levels over an extended period require higher drug concentrations and the use of lower dosage might contribute to resistance development.

c. The site-specificity of the drug delivery system at the adequate dosage and at the right time in a safe and reproducible manner [25].

d. Most of the drugs used are targeted towards infected erythrocytes, but due to amphiphilicity of drugs they are distributed into body tissues, get metabolized in the liver rapidly, and get associated with plasma proteins in blood vessels and the drug in the blood is taken out quickly from circulation resulting in shorter half-lives. Compensating for this higher dosage must be given leading to toxic effects and local concentrations of drugs contributing to drug resistance development. So, a higher degree of targeting is necessary.

e. Drugs targeting at multiple stages of the life cycle of malaria parasite are required.

f. For optimized drug therapy, new medicines must also be able to reduce transmission and prevent relapse of dormant forms.

g. The newer molecules must be screened to have selection wider for five species of the parasite which infects humans.

h. Risk assessment to resistance development keeping in mind the mechanisms of development of resistance should be done.

i. Affordable medicines should be produced and provided.

\section{1) Challenges faced by the eradication agenda:}

a. Single-dose cure leads to decreased treatment costs and observed administration increased course completions.

b. Safer drugs to be given that remain at the site of action for at least two parasite cycles. Activity should be against all species especially towards drug-resistant strains and the compound used should have very less selectivity for resistant phenotypes.

c. Transmission-blocking preventing transmission of parasites breaking the cycle, gametocyte stage specificity is a prerequisite.

d. Hypnozoites treatment and relapse prevention. Preventing relapses and getting drugs that reduce gametocyte formation or kill them are highly desirable but hardly explored domain.

e. Chemoprevention: RTS, S ('R' stands for the central repeat region of Plasmodium, ' $\mathrm{T}$ ' for the $\mathrm{T}$-cell epitopes circumsporozoite protein (CSP) and ' $\mathrm{S}$ ' stands for Hepatitis B surface antigen) vaccine is available for children for but, there is a significant need of a molecule effective for adult population. Natural immunity to the disease may fall in Africans and newer drugs with higher half-lives are required. Potent depot formulations with a daily human dosage of less than $10 \mathrm{mg}$ and preferably less than $1 \mathrm{mg}$ is required [26].

\section{CURRENTLY INVESTIGATED ANTIMALARIAL STRATEGIES}

\section{Transmission blocking agents:}

They target end points such as:

a. The mature gametocytes formed in the human host are completely and efficiently killed.

b. The development of gametocytes into ookinetes and then into sporozoites in the mosquito is inhibited. Tafenoquine, NITD609 and GNF156 has in vitro shown such activity. Tafenoquine is particularly effective in curing of relapsing malaria and prevention of $P$. vivax and $P$. falciparum infections. Other blocking agents are: Trioxaquine, U1302, Epoxomicine, Tipranavir (HIV protease inhibitor), Thiostrepton, Cycloheximide, and Ketotifen [27, 28].

\section{Phospholipid analogs:}

These are based on the mechanism of inhibition of the de novo biosynthesis pathway of phosphatidylcholine in plasmodium. The Phospholipid metabolism is absent in mature erythrocytes but is rapid in infected ones. Choline transport from serum to $\mathrm{RBC}$ is affected by erythrocyte choline carriers (ECC) and in infected ones, parasite encoded ECC is involved in offering another potential target to antimalarial drugs. Phospholipid analogs specifically accumulates in infected RBSs, blocking phosphatidylcholine biosynthesis and interacting with hemozoin. Some of the investigational agents are:

Mono-quaternary agents

Bis-thiozolium salts (albitiazolium) [29]

\section{Apicoplast targeting:}

Targeting is focused on apicoplast, an organelle ancestrally related to chloroplasts. The apicoplast is involved in a nonessential pathway in the asexual blood stages of Plasmodium but is having a key role in the development of infectious liverstage forms. Tetracycline is effective in such a targeting objective [30].

4. Natural Products:

Natural products and medicinal plants have been extensively evaluated and reported for therapies of many human diseases. Artemisinin and Quinine derivatives have been taken from nature's reserve. Malaria chemotherapy can be optimized by modifications in existing agents with natural products. Table 1 gives examples of natural products and extracts with confirmed or presumed activity in humans $[31,32,33]$. 


\section{Indo Global Journal of Pharmaceutical Sciences, 2021; 11(1): 15-27}

\begin{tabular}{|c|c|c|c|}
\hline S. No. & Natural drug & Source & Mechanism of action \\
\hline 1. & Quinine & Cinchona genus & $\begin{array}{l}\text { Assumed to be similar to chloroquine and prevent Heme } \\
\text { polymerisation }\end{array}$ \\
\hline 2. & $\begin{array}{l}\text { Lapachol } \\
\text { Lapinone } \\
\text { Atovaquon }\end{array}$ & Bignoniaceae & Electron transport inhibition \\
\hline 3. & Artemisinin & Artemisia Annua & $\begin{array}{l}\text { Free radical activation in the presence of free ferrous } \\
\text { Iron liberated in erythrocytes by parasite digestion of } \\
\text { Haemoglobin }\end{array}$ \\
\hline 4. & Yingzhaosu A & $\begin{array}{l}\text { Artabotrysuncinatus } \\
\text { (Ying Zhao) }\end{array}$ & $\begin{array}{l}\text { Presumed to be free radical activation in the presence of } \\
\text { free ferrous iron liberated in erythrocytes by parasite } \\
\text { digestion of haemoglobin }\end{array}$ \\
\hline 5 . & Cryptolepine & Cryptolepissanguinolenta & DNA intercalation \\
\hline 6. & Curcumin & Curcuma Longa & Antioxidant activity \\
\hline 7. & Gedunin & Azadirachtaindica & HSP90 inhibitor \\
\hline
\end{tabular}

Table 1: Natural products and extracts with confirmed or presumed activity in humans $[33,34,35]$ •

\begin{tabular}{|c|l|l|}
\hline S. No. & \multicolumn{1}{|c|}{ Drug } & \multicolumn{1}{|c|}{ Limitations } \\
\hline 1. & $\begin{array}{l}\text { Mefloquine (singly, combination } \\
\text { with Sulfapyrridine and } \\
\text { Artesunate) }\end{array}$ & Resistance, toxicity, neuropsychiatric adverse events \\
\hline 2. & Doxycycline(combination) & Non-compliance \\
\hline 3. & $\begin{array}{l}\text { Tefenoquine (singly \& } \\
\text { combination) }\end{array}$ & Haemolysis in patients with G6PD deficiency \\
\hline
\end{tabular}

Table 2: Drugs reconsidered for antimalarial therapy along with their limitations $[38,39,40,41,42,43]$.

\begin{tabular}{|c|l|rl|}
\hline S. No & \multicolumn{1}{|c|}{ Category } & \multicolumn{1}{c|}{ Examples } \\
\hline 1. & Anti-HIV & $\begin{array}{l}\text { Saquinavir, Ritonavir, and Indinavir are effective against } \\
\text { P. falciparum }\end{array}$ \\
& & $\begin{array}{l}\text { Act by inhibiting the parasite's aspartic proteases, termed } \\
\text { Plasmepsins, located on food/digestive vacuole or non- } \\
\text { digestive vacuole }\end{array}$ \\
\hline 2. & Anti-cancer & $\begin{array}{l}\text { SU-11274 and Bay 43-9006 } \\
\text { Targets plasmodium protein kinase }\end{array}$ \\
\hline
\end{tabular}

Table 3: Repurposing of drugs $[46,47,48,49,50,51]$ 
Indo Global Journal of Pharmaceutical Sciences, 2021; 11(1): 15-27

\begin{tabular}{|c|c|c|c|}
\hline S. No & $\begin{array}{c}\text { Types of } \\
\text { modification }\end{array}$ & Name of drug/ Chemical entity & Effect of modification \\
\hline 1. & Derivatization & $\begin{array}{l}\text { - } \\
\text { 4-position modified quinoline- } \\
\text { methanol compounds } \\
\text { (Mefloquine) } \\
\text { - } \quad \text { 8-aminoquinoline analogs } \\
\text { - }\end{array}$ & $\begin{array}{l}\text { - } \quad \text { Less toxicity } \\
\text { - } \quad \begin{array}{l}\text { Better pharmacological and } \\
\text { toxicological profile } \\
\text { - Water solubility }\end{array} \\
\end{array}$ \\
\hline 2. & Hybridization & $\begin{array}{ll}\text { - } & \text { 4-amino-7-chloroquinoline } \\
\text { antimalarial } \\
\text { - } & \text { DHA+carboxylic acid } \\
\text { - Trioxaquines }\end{array}$ & $\begin{array}{ll}\text { - } & \text { Active against } \\
\text { chloroquineresistantstrains } \\
\text { - } \\
\text { Improved antimalarial } \\
\text { activity }\end{array}$ \\
\hline
\end{tabular}

Table 4: Modifying existing compounds [52]

\section{Hybrid compounds:}

Researchers have combined Chloroquine with compounds reverting or blocking the resistance mechanisms. These are as follows:

a. Imipramine (Antidepressant) - has shown mild anti-malarial activity and inhibition of $\mathrm{P}$. falciparum chloroquine resistance transport responsible for chloroquine efflux in chloroquineresistant parasites.

b. Astemizole (Antihistamine): has shown chloroquine resistance reversal activity [34].

\section{Combination antimalarial drugs:}

They allow multidrug and multi-kinetic drug delivery systems. Modules or release units are having alternating concave and convex bases to obtain interlocked assembly. The modules can be formulated for immediate or prolonged release thus providing flexibility in programming. The alteration of an assemblage of modules can help is required pharmacokinetics of the selected drugs, release programs, site and time of delivery of single drugs [35].

\section{NEXT GENERATION ANTIMALARIAL TREATMENTS}

These are as follows:

1. Reconsidering drugs with limitations

2. Repurposing of drugs

3.Development of new drugs by modifying existing compounds

4. Creating new combinations

5. Anti-sequestration compounds an interesting possibility

6. Novel routes of drug delivery

7. Drug Delivery to the malaria parasite using an arterolanelike scaffold

8. Targeting vitamin biosynthesis pathways

9. Multiple targeting approaches

10. Nanotechnology-based approaches in anti-malarial therapy

\section{Reconsidering drugs with limitations:}

Following drugs have been reconsidered for antimalarial therapy due to reasons: Refer Table 2: Drugs reconsidered for antimalarial therapy along with their limitations [36,37, 38, 39, 40, 42].

\section{Repurposing of drugs:}

One of the approaches for rapidly identifying and bringing new drugs to the market for the treatment of malaria is to repurpose existing drugs that are currently approved for the treatment of other infections or diseases. Refer Table 3: Repurposing of drugs [43, 44, 45, 46, 47, 48].

\section{Development of new drugs by modifying existing compounds:}

Refer Table 4 for examples of Modifying existing compounds [49].

\section{Optimizing available antimalarial:}

This is based on the understanding that the drug-resistant mutants are less likely to fit and survive than their wild-type strains in the absence of drugs they have developed resistance [50]. For example, Chloroquine was removed from Malawi, a region with a high level of Chloroquine resistance, Chloroquine sensitivity returned [51].

\section{Creating new combinations:}

New combinations of drugs for increasing the effectiveness of malaria therapy have been investigated. Few of them with some success are as follows:

a. Chlorpheniramine and Chloroquine

b. Primaquine and Antiretroviral protease inhibitors (Saquinavir)-Chloroquine resistance reversal agents

c. Fluoxetine (antidepressant) along with Chloroquine and Mefloquine- resistance reversal agents

d. Ketoconazole (antifungal) along with Mefloquine-resistance reversal agents [52]. 


\section{Indo Global Journal of Pharmaceutical Sciences, 2021; 11(1): 15-27}

\section{Anti-sequestration possibility: \\ compounds-an interesting}

These agents not only kill the malaria parasite but also prevent them from causing severe forms of disease by inhibiting the parasite's ability to sequester. Sequestration is due to parasitederived adhesions, expressed on the surface of mature infected erythrocytes, binds to receptors on human cells (cytoadhesion). Organ-specific sequestration of parasite results in cerebral and placental malaria [53]. Cytoadhesion has been inhibited by the usage of antiretroviral protease inhibitors (Ritonavir and Saquinavir) and seaweed-derived polysaccharides (Carrageenan's) in in-vitro setting. Levamisole used in adjunctive therapy along with quinine and Doxycycline has been found active for the anti-sequestering activity.

\section{Novel routes of drug delivery:}

while being used as a general rule, oral for uncomplicated malaria and Parenteral for severe and complicated malaria it has been found that novel routes are having improved specificity, efficacy, tolerability and therapeutic index of existing drugs.

a. Ceramic implants: Polymers (polylactic acid, gelatin or chitosan) are matrices for ceramic particles that can introduce a tailored biodegradable drug. Gelatin ceramic implants have been reported to sustain consistently therapeutic blood Chloroquine (CQ) concentrations in experimental animals.

b. Transdermal route of drug delivery: The advantages of Transdermal route includes- avoidance of hepatic first-pass metabolism, easy administration and possibility of immediate withdrawal of the treatment and sustained plasma drug concentration.

c. Rectal route: Provides improved parasitological efficacy and pharmacokinetics in children with P. falciparum malaria relative to intramuscular and intravenous injections. Rectal administration is a painless procedure that enables selfadministration and reduces the risk of infections from already used needles.

d. Nasal delivery: One advantage of the nasal route is the simplicity of administration and allowing easy treatment following the first signs of illness. Moreover, the nasal cavity allows the drugs to be delivered directly to the brain via the nasal membrane, which is vital in cerebral malaria [54].

\section{Drug Delivery to the Malaria Parasite Using an Arterolane-Like Scaffold:}

Artemisinin and Arterolane act via initial reduction of peroxide bond in a process mediated by ferrous iron sources in the parasite. Arterolane -like 1, 2, 4-trioxolanes can be used for releasing a tethered drug species within the parasite. They provide the advantage of significantly low molecular weight and superior metabolic stability [55].

\section{Targeting vitamin biosynthesis pathways:}

Biosynthesis pathways for vitamins B6 (pyridoxine) and B1 (thiamine) have been identified in plasmodium and analyzed for their suitability as new potential drug targets of new drug discoveries [56].

\section{Multiple stages targeting approach:}

Extensive research is undergoing to find or develop drugs targeting multiple plasmodium life stages thus increasing the effectiveness of drug therapy. Two under investigation are:

1. Spiroindoles

2. Imidazolopiperazines [57]

\section{NANOTECHNOLOGY BASED ANTIMALARIAL APPROACH}

Role of nanotechnology in diagnosis

Role of nanotechnology in malaria therapy

Gene delivery with nanomaterials for antimalarial treatment Malaria vaccination: exploring nanotechnology

\section{A. Role of nanotechnology in diagnosis:}

1. Nanosomic DNA analyzer: A smartphone-like device which can analyze malaria strain DNA from a finger-prick of blood has been developed which enables a personalized prescription of the drug combination to be given to the patient straight [58].

2. Vapor nano bubbles: It uses a low powered laser that creates tiny vapor "Nano bubbles" inside malaria infected cells that gives out a unique acoustic during bursting to provide a very sensitive diagnosis $[59,60]$.

3. Detection and quantification of malaria parasite infection: Microscopically two methods are used for quantifying the infection:

i) Giemsa staining-identifying ring and schizont stages of malaria parasite development and infected cells to noninfected cells.

ii) Fluorescent staining with SYBR green-to identifies malaria-infected cells, specific stages of the parasite development and to identify viable parasites $[61,62,63]$.

4. Flow strip chip for diagnosis of malaria infection: It is based on the development of the micro-fluidic channels which mimic the sizes and shapes of capillary blood vessels and help in observing host-parasite interaction and malaria-infected red blood cells in the capillary environment. This has the potential for more accurate diagnosis particularly, at field sites [64].

5. Quantum dots: these can be used sometimes by studying the delivery of the contents of liposomes to erythrocytes infected by malaria parasite. 


\section{Indo Global Journal of Pharmaceutical Sciences, 2021; 11(1): 15-27}

\section{B. Role of nanotechnology in malaria therapy:}

Nano particulate drug delivery systems are promising to malaria therapy due to their ability to alter biopharmaceutics and pharmacokinetic property of the drug molecule. They can remain in blood stream for longer period of time resulting in longer interaction time between the system and infected cells. Other advantages are protection of unstable drugs, cell adhesion properties, ability to be modified (generally surface) to increase targeting abilities, etc. These can be used sometimes by studying the delivery of the contents of liposomes to erythrocytes infected by malaria parasite [65].

\section{Liposomes as Nanocarriers for antimalarial targeting:}

\subsection{Conventional:}

1. Long-circulating neutral liposomes increase oral bioavailability and intravenously increased half-life of drug.

2. Long-circulating negatively charged liposomes show an increase in therapeutic and prophylactic potential $[66,67,68]$.

\subsection{Targeted:}

1. Peptide targeted liposomes: The peptide is chemically bound to surface of PEGylated liposomes prepared with labeled lipids and has shown 100 times increase in targeting to hepatocytes and non-parenchymal liver cells than to other organs (heart, lungs \& kidney) and 10 times more than to spleen $[69,70]$.

2. Antibody bearing liposomes: Antibody-targeted liposomes have shown to induce considerable enhancement in the liposome binding to erythrocytes in vivo finally resulting in reduced toxicity, improved therapeutic efficacy against malaria parasites, modified pharmacokinetics, and ensured prolonged in vivo release of drugs [71].

\subsection{Liposomes as adjuvants for malaria vaccines:}

Liposome-based vaccines have shown the potential to induce both antibody and cellular immune response by simultaneously activating the major histocompatibility complex (MHC) class I and MHC class II pathways [72, 73].

\section{Solid lipid nanoparticles:}

Transferrin-loaded nanoparticles have shown to have significantly enhanced the brain localization of quinine compared with unconjugated SLN or drug solution and reduced the amount of drug reaching the liver. Curcumin loaded nanoparticles or herbal solid lipid nanoparticles loaded into preformed chitosan nanoparticles obtained by ionotropic gelation with tripolyphosphate has enabled improved drug stability and enhanced drug oral bioavailability when administered to mice infected with a lethal strain of P. yoelii resulting in prolonging the survival of treated mice [74]. Chitosan-based Nano drug delivery system has shown overcome the efflux of CQ from DV and as well as reducing the resistant zones of CQ as well as CS-TPP nanoparticle conjugated chloroquine. The resistant zone of the age-old CQ as well as CS-TPP nanoparticle conjugated chloroquine [75].

2. Nanoject: The smaller NLC's extravagate faster to the blood, thus improving pharmacological effect and increasing duration of action of drugs like Artemether with very short half-life [76].

3. Nano and micro emulsions: The Nanoemulsions (NE), oilin-water $(\mathrm{O} / \mathrm{W})$ emulsions with a mean diameter of $\leq 500 \mathrm{~nm}$ have advantages of Low cost and availability as oral dosage forms [77].

4. Micelle: Pharmaceuticals like micelles are providing new and exciting perspectives in the clinical treatment of several severe diseases, such as cancer, infections or neurodegenerative diseases $[78,79]$.

\section{POLYMER BASED CARRIERS}

1. Polymeric nanoparticles: They can be modulated for both active and passive targeting and possess high stability in biological fluids and under stress conditions of preparation and storage, which qualifies them as promising drug delivery systems for antimalarial. PLGA-nanoparticles loaded with monensin loaded have been found to be 10 times more effective in inhibiting the growth of P. falciparum in vitro as compared to free monensin with efficacy depending upon the molecular weight of the polymer $[80,81]$.

2. Dendrimers: Hydrophobic antimalarial drugs solubilized in dendrimers have shown long circulating and site-specific characteristics. PEG-lysine type dendrimer along with chondroitin-sulfate A (CSA) has shown sustained and controlled delivery of the drug via the intravenous route of administration [82, 83, 84].

3. Cyclodextrin and inclusion complexes with antimalarial drugs: It has been suggested that sulphated-CDs inhibit Plasmodium growth by interacting with the anion transport protein, AE1. Furthermore, sulphated-CDs were found to be active against $P$. falciparum cultures and to inhibit $P$. berghei merozoite entry in RBC [85].

4. Nanosuspensions: Nanosuspensions are sub-micron colloidal dispersions of a pure poorly water-soluble drug stabilized by surfactants, without any matrix material suspended in dispersion and have been shown to be active in malaria drug therapy $[86,87]$.

5. Nanocapsules: They show high entrapment efficiencies of lipophilic drugs and their low polymer content, low inherent toxicity are some of the main advantages of $\mathrm{NC}$ along with some therapeutic advantages of solubilization of poorly watersoluble drugs, protection against drug inactivation in the gastrointestinal tract, protection against drug toxicity enhanced 


\section{Indo Global Journal of Pharmaceutical Sciences, 2021; 11(1): 15-27}

permeation of drugs through mucosal surfaces and prolongation of the blood circulation of injected drugs $[88,89$, 90, 91, 92].

6. Self-emulsifying drug delivery systems (SMEDDS): is one of the drug delivery systems that use a microemulsion achieved by chemical rather than mechanical means and have shown the better efficacy of Artemether when administered as Artemisinin suppository in terms of rapid parasites clearance and safety. The in vivo antimalarial activity showed a significant increase in antimalarial activity compared to Larither both in terms of survival and parasitemia progression. There is an increase in solubility and stability can be given orally, less costly and is patient-friendly [93]. Selfnanoemulsifying drug delivery systems showed increased oral bioavailability and can be used along with other conventional available drugs and possibly be used for Artemisinin based combination therapy [94].

7. Liquid crystal nanoparticles: for solubility enhancement of antimalarial drugs [95].

8. Pheroids: are colloidal delivery systems that contain stable lipid-based submicron and micron-sized structures and these structures can be uniformly dispersed in colloidal systems. They can be used to entrap, transport and deliver the therapeutics to a specific organ or part of the body and since they are made up of naturally occurring compounds, they are biocompatible, biodegradable and safe to be used in medicine. This technology has been used to deliver the antimalarial drugs Artemisone and artemiside which are derivatives of Artemisinin with good antimalarial activity but poor and erratic absorption upon oral administration [96].

9. Agglomerates: antimalarial drugs could be formulated as agglomerates and blended to obtain a combination product. Another option could be the mixing of two or more antimalarial drug powders before agglomeration with the goal to create multidrug antimalarial agglomerates. They are easy to handle, but at the same time the agglomerate structure preserves the advantages of a micronized dosage form with respect to biopharmaceutics and pharmacokinetics. This product appears to be particularly suitable for use in children and the elderly

\section{OTHER ANTIMALARIAL STRATEGIES:}

1. Nanoerythrosomes: These ensure delayed drug release as well as higher drug loading capacity [97].

2. Ferrous iron-dependent drug delivery: Malaria parasites produce high concentrations of mobile ferrous iron as a consequence of their catabolism of host hemoglobin in the infected erythrocyte. By using activity-based probes, successfully the Fe-II-dependent and parasite-selective delivery of a potent dipeptidyl aminopeptidase inhibitor has been successfully demonstrated [98].
3. Resealed erythrocytes: They have the ability to selectively accumulate within RES organs thus making them a useful tool during the delivery of anti-parasitic agent specially the parasitic diseases that involve harboring parasites in the RES organs. Results have been found to be positive [99].

\section{Gene delivery with nanomaterials for antimalarial treatment:}

The aim of using the antisense approach is to interfere with gene expression by preventing the translation of proteins from mRNA. As the malaria parasite divides rapidly after infection of human erythrocytes, DNA-replicating, enzyme-related genes, such as those of topoisomerases offer promising targets in the parasite.

Another study reported the use of antisense oligonucleotides (ODNs) against malarial topoisomerase II gene incorporated in chitosan nanoparticles [100].

\section{Malaria vaccination: exploring nanotechnology:}

Plasmid DNA encoding for a fragment of the merozoite surface protein MP1 was condensed on the surface of polyethylene mine-coated superparamagnetic iron oxide nanoparticles (PEI-SPIONs). Due to magnetic transfection, the magnetic Nano carriers have been proven to improve efficiency and rate of gene delivery to different tissues. The DNA/PEI-SPIONs complexes administered subcutaneously, intramuscularly or intra-peritoneal elicited $\operatorname{IgG} 2 \mathrm{a}$ and $\operatorname{IgG} 1$ responses involved in the protective immunity against malaria [101].

\section{OTHER DEVELOPMENTS IN ANTIMALARIAL THERAPY}

1. Calcineurin inhibitors:

Harvard T.H. Chan School of Public Health researchers have found that a malaria parasite protein called calcineurin is essential for parasite invasion into red blood cells. The new findings suggest that parasite calcineurin should be a focus for the development of new antimalarial drugs. They found that the protein allows the malaria parasite in recognizing and attaching to the surface of red blood surface. Parasites with inhibited calcineurin showed ineffectiveness due to failure to invade [102].

\section{Novel Drug delivery to the mosquito:}

This is based on administering anti-malarial to humans with sufficiently log half-life to increase their probability of being picked up by anopheles and good gametocidal activity showing agent may increase effectiveness [103].

3. Anti-malaria tea: It has been found that many communities in Asia and Africa drink tea made from Chinese sweet wormwood plant named Artemesia to help stave off 


\section{Indo Global Journal of Pharmaceutical Sciences, 2021; 11(1): 15-27}

malaria as the plant is found to be source of Artemisinin derivative with other drugs. However, its bitter taste and risk of resistance development remains major challenge towards its use in mass control strategies against malaria [104].

4. Normal blood cell as carriers: They ensure that the parasite doesn't even get the chance to start its development cycle, which it normally does during a 'recovery period' after a quick and stealthy entry into an RBC. They also have advantages of being biocompatible, long life span and available safe mechanisms for elimination from the body [105].

\section{Plasmepsins:}

The parasitic aspartic proteases are termed as Plasmepsins (Plms) that are involved in the hemoglobin catabolism that occurs during the erythrocyte stage of the malaria parasite life cycle. They have provided new potential targets of antimalarial drugs [106].

\section{Magnetic-resonance method:}

This method works by applying a mechanical torque through magnetic field to the hemozoin in the malaria parasite to get the iron crystals to rotate, oscillate, agitate, vibrate, and churn achieving the destruction of the parasite, either directly by mechanical battery that can tear the parasite's internal membranes or indirectly by conversion of the mechanical energy into heat [107].

7. Enzyme targeting: The new anti- malaria drug interferes with parasite histone methyltransferases, enzymes which are crucial to the parasite's growth and viability during the blood stage of its lifecycle rapidly killing parasites in culture and greatly reduce parasite infection in mice in a single day [108].

8.Protein targeting: Targeting the protein responsible for making environment inside the red blood cell appropriate for the parasite to survive inside the cell is another novel approach [109].

9. Dual acting drug: A molecule identified and called as DSM265, targets parasite in both liver and blood stages and remains in the body for an extended period of time. This has the potential to act as both treatment and prevention for the disease [110].

10. ART hybrid: The ART hybrid, 1,2,4,5-tetraoxane (RKA 182), is considered to be an outstanding antimalarial candidate with the advantages of enhanced stability, lower toxicity and improved biopharmaceutical properties (ADME) compared to the semi-synthetic antimalarial ART.

\section{Hybrid molecule:}

The hybrid molecule MEFAS, which is derived from MQ and AS, is more effective and less toxic than the combination of MQ and AS, as shown using an experimental in vitro test with P. falciparum and in animal models. The hybrid compound comprises dual functions) [111].
12. Ferroquine: A chloroquine analogue is considered to be the most advanced organometallic antimalarial is in clinical trials for the treatment of uncomplicated malaria[112].

\section{CONCLUSION}

There are a large number of opportunities present for increasing the efficacy of antimalarial therapy. The newer targets for antimalarial drugs have provided a large number of opportunities as well as newer approaches to attack multiple stages have further boosted the fight against the parasitic disease. Newer molecules with increased efficacy, target specificity and other properties have provided a new hope as per as challenges related to antimalarial therapy and eradication agenda is concerned. Pharmaceutical technology can provide unlimited opportunities for improving the efficacy of the currently used antimalarial drugs and the new chemical entities characterized by poor solubility, chemical instability, inadequate bioavailability profile and toxicity. The wide range of modifications of the surface properties of these nanometric carriers are aimed at improving antimalarial selectivity in the newly discovered parasite targets have been very less exploited to date. Furthermore, there are several newer approaches which are under investigation. It is expected that this review will contribute towards better understanding of malaria disease and provide an overview of newer strategies for developing smart, well-tolerated cost-effective and targeted therapeutic nanosystems to treat patients infected with malaria.

\section{CONFLICTS OF INTEREST}

The authors declare that there is no conflict of interest regarding the publication of this paper.

\section{ACKNOWLEDGEMENT}

None

\section{DATA AVAILABILITY}

Not declared.

\section{FUNDING SOURCE}

Not declared

\section{REFERENCES}

1. World Malaria Report 2008 https://www.who.int/malaria/publications/atoz/9789241563697/en/.

(Accessed on: 1st April, 2011)

2. Carter, R., Mendis, K.N. Evolutionary and historical aspects of the burden of malaria. Clin. Microbiol., Rev. 2002; 564594. 


\section{Indo Global Journal of Pharmaceutical Sciences, 2021; 11(1): 15-27}

3. White, N. Antimalarial drug resistance and combination chemotherapy. Philos. Trans. R. Soc. Lond. B Biol. Sci., 1999; 739749 .

4. Nel, A., Xia, T., Mädler, L. Li N. Toxic potential of materials at the nano level. Science 2006; 311: 622-627.

5. Sinka, M.E., Bangs, M.J., Manguin S. A global map of dominant malaria vectors. Parasite Vectors., 2012; 5: 69.

6. White, N.J. et al. Malaria. 2013

7. Gething, P.W., Patil, A.P., Smith, D.L. A new world malaria map: Plasmodium falciparum endemicity in 2010. Malaria J., 2011; 10: 378 .

8. White, N.J. et al. Malaria. 2013.

9. Cooke, B., Coppel, R., Wahlgren, M. Falciparum malaria: sticking up, standing out and outstanding. Parasitology Today., 2000; 16: 416-20.

10. Pain, A., Ferguson, D.J., Kai, O. Platelet-mediated clumping of Plasmodium falciparum-infected erythrocytes is a common adhesive phenotype and is associated with severe malaria. Proc. Natl. Acad. Sci USA., 2001; 98: 1805-10.

11. Doumbo, O.K., Thera, M.A., Kone, A.K. High levels of Plasmodium falciparum rosetting in all clinical forms of severe malaria in African children. Am J Trop Med Hyg., 2009; 81: 987-93.

12. Pongponratn, E., Turner, G.D., Day, N.P. An ultrastructural study of the brain in fatal Plasmodium falciparum malaria. Am J Trop Med Hyg., 2003; 69: 345-59.

13. Greenwood, B.M. et al. Malaria: Progress, perils, and prospects for eradication. J. Clin. Invest., 2008; 118: 1266-1276.

14. Krishna, S., Bustamante, L., Haynes, R.K., Staines, H.M. Artemisinins: their growing importance in medicine. Trends Pharmacol. Sci., 2008; 29: 520-527.

15. Biagini, G.A., Ward, S.A., Bray, P.G. Malaria parasite transporters as a drug-delivery strategy. Trends Parasitology., 2005; 21: $299-301$

16. Magalhães, N.S.S., Mosqueira, V.C.S. Nanotechnology applied to the treatment of malaria. Advanced drug delivery reviews., 2010; 62: 560-575.

17. Wernsdorfer, W.H., Payne, D. The dynamics of drug resistance in Plasmodium falciparum. Pharmacol. Ther., 1991; 50; 95-121.

18. Hayton, K., Su, X.Z. Genetic and biochemical aspects of drug resistance in malaria parasites. Curr. Drug Targets Infect. Disord., 2004; 4: 1-10.

19. Hyde, J.E. Drug-resistant malaria - an insight. FEBS J., 2007; 274: 4688-4698.

20. Ekland, E.H., Fidock, D.A. Advances in understanding the genetic basis of antimalarial drug resistance. Curr. Opin Microbiol., 2007; 10: 363-370.

21. Kidgell, C., Winzeler, E.A. Using the genome to dissect the molecular basis of drug resistance. Future Microbiol., 2006; 1: 185-199.

22. Cui, L., Su, X.Z. Discovery, mechanisms of action and combination therapy of artemisinin. Expert Rev. Anti-Infect Ther., 2009; 7: 999-1013.

23. O'Neill, P.M., Barton, V.E., Ward, S.A. The molecular mechanism of action of artemisinin-The debate continues. Molecules., 2010; 15: 1705-1721.
24. Sinha, S., Medhi, B., Sehgal, R. Challenges of drugresistant malaria. Parasite Journal., 2014;21-60.

25. Patricia, U., Joan, E., Alberto, A., Alfred, C. Study of the efficacy of antimalarial drugs delivered inside targeted immunoliposomal nanovectors. 2011; 6:620.

26. Newton, C.R.J.C., Krishna, S. Severe falciparum malaria in children: Current understanding of path physiology and supportive treatment. Pharmacol. Ther., 1998; 79: 1-53.

27. Mishra, S.K., Newton, C.R. Diagnosis and management of the neurological complications of falciparum malaria. Nat. Rev. Neurol., 2009; 5:189-198.

28. Peyrottes, S., Caldarell, S., Wei, S., Pellet, C.P.A., Vial, H. Choline Analogues in Malaria Chemotherapy. Current Pharmaceutical Design., 2012; 18: 3454-3466.

29. Erica L. D. et al. Tetracycline Specifically Target the Apicoplast of the Malaria Parasite Plasmodium falciparum; Antimicrobial agents and chemotherapy., 2006; 3124-3131.

30. Wells, T.N.C. Natural products as starting points for future anti-malarial therapies: going back to our roots? Malaria Journal., $2011 ; 10$ (1)

31. Lisgarten, J.N., Coll, M., Portugal, J., Wright, C.W., Aymami, J. The antimalarial and cytotoxic drug cryptolepine intercalates into DNA at cytosine-cytosine sites. Nat Struct. Biol., 2001; 9:57-60.

32. Hieronymus, H.et al. Gene expression signature based chemical genomic prediction identifies a novel class of Hsp90 pathway modifiers. Cancer Cell., 2006; 10:321-330.

33. Oliveira, R. et al. From hybrid compounds to targeted drug delivery in antimalarial. Bioorganic \& Medicinal Chemistry., 2015; 15; 23(16):5120-30.

34. Losi, E. et al. Assemblage of novel release modules for the development of adaptable drug delivery systems. J. Control. Release., 2006; 111: 212-218.

35. Losi, E. et al. Assemblage of novel release modules for the development of adaptable drug delivery systems. J. Control. Release., 2006; 111: 212-218.

36. Price, R.N. et al. World Antimalarial Resistance Network: Clinical efficacy of antimalarial drugs. Malar. J., 2007; 6:119.

37. Schlagenhauf, P., Adamcova, M., Regep, L., Schaerer, M.T., Rhein, H.G. The position of mefloquine as a 21 st century malaria chemoprophylaxis. Malar. J., 2010; 9: 357.

38. Dow, G.S. et al. Transcriptional profiling of mefloquineinduced disruption of calcium homeostasis in neurons in vitro. Genomics., 2005; 86: 539-550.

39. Dow, G.et al. Mefloquine induces dose-related neurological effects in a rat model. Agents Chemother., 006:1045-1053.

40. Nevin, R.L., Pietrusiak, P.P., Caci, J.B. Prevalence of contraindications to mefloquine use among USA military personnel deployed to Afghanistan. Malar. J., 2008; 7: 30.

41. Policy memorandum on the use of mefloquine in malaria prophylaxis; http://www.lariaminfo.org/pdfs/policy-memo-secydefense\%20malaria prophylaxis.pdf (accessed 1 April 2011).

42. Shanks, G.D. A new primaquine analogue, tafenoquine (WR 238605), for prophylaxis against Plasmodium falciparum malaria. Clin. Infect. Dis., 2001; 33: 1968-1974.

43. Walsh, D.S. Randomized dose-ranging study of the safety and efficacy of WR 238605 (Tafenoquine) in the prevention of 


\section{Indo Global Journal of Pharmaceutical Sciences, 2021; 11(1): 15-27}

relapse of Plasmodium vivax malaria in Thailand. J. Infect. Dis., 1999; 180: 1282-1287.

44. Andrews, KT. Potencies of human immunodeficiency virus protease inhibitors in vitro against Plasmodium falciparum and in vivo against murine malaria. Antimicrob. Agents Chemother., 2006; 50: 639-648.

45. Parikh, S., Liu, J., Sijwali, P., Gut, J., Goldberg, D.E., Rosenthal PJ. Antimalarial effects of human immunodeficiency virus type 1 protease inhibitors differ from those of the aspartic protease inhibitor pepstatin. Antimicrob. Agents Chemother., 2006; 50: $2207-$ 2209.

46. Skinner-Adams, T.S., Andrews, K.T., Melville, L., McCarthy, J., Gardiner, D.L. Synergistic interactions of the antiretroviral protease inhibitors saquinavir and ritonavir with chloroquine and mefloquine against Plasmodium falciparum in vitro. Antimicrob. Agents Chemother., 2007; 51:759-762.

47. Sattler, M.A. novel small molecule met inhibitor induces apoptosis in cells transformed by the oncogenic TPR-MET tyrosine kinase. Cancer Res., 2003; 63: 5462-5469.

48. Doerig, C. Malaria: Targeting parasite and host cell kinomes. Biochim. Biophys. Acta., 2010; 1804: 604-612.

49. Jirage, D., Keenan, S.M., Waters, N.C. Exploring novel targets for antimalarial drug discovery: Plasmodial protein kinases. Infect. Disord. Drug Targets., 2010; 10: 134-146.

50. Walliker, D., Hunt, P., Babiker, H. Fitness of drug-resistant malaria parasites. Acta Trop., 2005; 94: 251-259.

51. Zhou, Z. et al. Decline in sulfadoxine-pyrimethamineresistant alleles after change in drug policy in the Amazon region of Peru. Antimicrob. Agents Chemother., 2008; 52: 739-741.

52. Egan, T.J., Kaschula, C.H. Strategies to reverse drug resistance in malaria. Curr. Opin. Infect. Dis., 2007; 20: 598-604.

53. Rowe, J.A., Claessens, A., Corrigan, R.A., Arman, M. Adhesion of Plasmodium falciparum-infected erythrocytes to human cells: molecular mechanisms and therapeutic implications. Expert Rev. Mol. Med., 2009; 1: 16.

54. Rogerson, S.J. Malaria in pregnancy and the newborn. Adv. Exp. Med. Biol., 2010; 659: 139-152.

55. Schneeweis, A., Muller-Goymann, C.C. Controlled release of solid-reversed micellar-solution (SRMS) suppositories containing metoclopramide-HCl. Int J Pharm., 2000; 196:193-196.

56. Fontaine, S.D. et al. Drug Delivery to the Malaria Parasite Using an Arterolane-Like Scaffold., 2015;10(1):47-51.

57. Isolmar, Schettert, Carsten, Wrenger. Targeting the vitamin biosynthesis pathways for the treatment of malaria., 2013; 5(7):76979.

58. $\mathrm{Wu}, \mathrm{T}$. et al. Imidazolopiperazines: Hit to Lead Optimization of New Antimalarial Agents. J. Med. Chem., 2011; 54 (14):5116-5130.

59. Vapor nanobubbles detect malaria through skin. https://www.futurity.org/vapor-nanobubbles-detect-malaria-skin/.

(Accessed on: 15th May 2011).

60. Vapor nanobubbles rapidly detect malaria through the skin https://www.chron.com/neighborhood/article/Vapor-nanobubblesrapidly-detect-malaria-through-9895552.php. (Accessed on: 15th May 2011).
61. Hleb, L. Hemozoin-generated vapour nanobubbles for transdermal reagent- and needle-free detection of malaria; PNAS., 2014; 111: 900-905.

62. Guy, R., Liu, P., Pennefather, P., Crandall, I. The use of fluorescence enhancement to improve the microscopic diagnosis of falciparum malaria; Malar J., 2007;6: 89.

63. Gligorijevic, B., Purdy, K., Elliott, D.A., Cooper, R.A., Roepe, P.D. Stage independent chloroquine resistance and chloroquine toxicity revealed via spinning disk confocal microscopy. Mol. Biochem. Parasitol., 2008; 589: 7-23.

64. Karl, S., Wong, R.P., St. Pierre, T.G., Davis, T.M., A comparative study of a flow cytometry- based assessment of in vitro Plasmodium falciparum drug sensitivity; Malara J., 2009; 8: 294.

65. Antia, M., Herricks, T., Rathod, P. K. Microfluidic modelling of cell-cell interactions in malaria pathogenesis. PLoS Pathog., 2007; 3: 939-948.

66. Alba, Pujol. Application of Quantum Dots to the Study of Liposome Targeting in Leishmaniasis and Malaria. International Journal of Theoretical and Applied Nanotechnology., 2014; 1-8.

67. Pirson, P., Steiger, R.F., Trouet A. Primaquine liposomes in the chemotherapy of experimental murine malaria. Ann. Trop. Med. Parasitol., 1980; 74: 383-391.

68. Pirson, P., Steiger, R.F., Trouet, A. The disposition of free and liposomally encapsulated antimalarial primaquine in mice. Biochem. Pharmacol., 1982; 31: 3501-3507.

69. Peeters, P.A.M., Leest, K.D., Eling, W.M.C., Crommelin. D.J.A. Chloroquine blood levels after administration of the liposome encapsulated drug in relation to therapy of murine malaria. Pharm. Res., 1989; 6: 787-793.

70. Haynes, S.M., Longmuir, K.J., Robertson, R.T., Baratta, J.L., Waring, A.J. Liposomal polyethylene glycol and polyethylene glycol-peptide combinations for active targeting to lier in vivo. Drug Deliv., 2008; 15: 207-217.

71. Agrawal, A.K., Gupta, C.M. Tuftsin-bearing liposomes in treatment of macrophage-based infections. Adv. Drug Deliv., Rev.2000; 41: 135-146.

72. Alving, C.R. Design and selection of vaccine adjuvants: animal models and human trials. Vaccine., 2002; 20 (Suppl. 3): S56S64.

73. Tyagi, R.K., Sharma, P.K., Vyas, S.P., Mehta, A. Various carrier system(s) mediated genetic vaccination strategies against malaria. Expert Rev. Vaccines., 2008; 7: 499-520.

74. Akhtar, F., Rizvi, M.M., Kar, S. Oral delivery of curcumin bound to chitosan nanoparticles cured Plasmodium yoelii infected mice. Biotechnol. Adv., 2012; 30: 310-320.

75. Tripathya, S. A novel chitosan based antimalarial drug delivery against Plasmodium berghei infection. Actatropica., 2013;128(3):494-503.

76. Joshi, M., Pathak, S., Sharma, S., Patravale, V. Design and in vivo pharmacodynamic evaluation of nanostructured lipid carriers for parenteral delivery of artemether. International Journal of Pharmaceutics., 2008; 364: 119-126.

77. Dierling, A.M., Cui, Z. Targeting primaquine into liver using chylomicron emulsions for potential vivax malaria therapy. Int. J. Pharm., 2005; 303: 143-152. 


\section{Indo Global Journal of Pharmaceutical Sciences, 2021; 11(1): 15-27}

78. Singh, K.K., Vingkar, S.K. Formulation, antimalarial activity and biodistribution of oral lipid nanoemulsion of primaquine. Int. J. Pharm., 2008; 347:136-143.

79. Couvreur, P., Vauthier, C. Nanotechnology: intelligent design to treat complex disease. Pharm. Res., 2006; 23:1417-1450.

80. Irache, J.M., Esparza, I., Gamazo, C., Agüeros, M., Espuelas, S. Nanomedicine: novel approaches in human and veterinary therapeutics. Vet. Parasitol., 2011; 180: 47-71.

81. Xu, T., Zhang, N., Nichols, H.L., Shi, D., Wen, X. Modification of nanostructured materials for biomedical applications. Mater. Sci. Eng., 2007; C27: 579-594.

82. Labhasetwar. V.D., Dorle, A.K. Nanoparticles: a colloidal drug delivery system for primaquine and metronidazole. J. Control. Release 1990; 18: 113-119.

83. Bhadra, D., Bhadra, S., Jain, N.K. PEGylated peptide-based dendritic nanoparticulate systems for delivery of artemether. J. Drug Deliv. Sci. Technol., 2005; 15: 65-73.

84. Bhadra, D., Bhadra, S., Jain, N.K. PEGylated peptide dendrimeric carriers for the delivery of antimalarial drug chloroquine phosphate. Pharm. Res., 2006; 23: 623-633.

85. Bhadra, D., Yadav, A.K., Bhadra, S., Jain, N.K. Glycodendrimeric nanoparticulate carriers of primaquine phosphate for liver targeting. Int. J. Pharm., 2005; 295: 221-233.

86. Crandall, I.E. et al. Sulfated cyclodextrins inhibit the entry of Plasmodium into red blood cells implications for malarial therapy. Biochem. Pharmacol., 2007; 73: 632-642.

87. Müller, R.H., Gohla, S., Dingler, A., Schneppe, T. Large scale production of solid lipid nanoparticles (SLNTM) and nanosuspensions (DissoCubes ${ }^{\mathrm{TM}}$ ) D. Wise (Ed.). Handbook of Pharmaceutical Controlled Release Technology, Marcel Dekker; New York., 2000; 359-376.

88. Patravale, V.B., Date, A.A., Kulkarni, R.M. Nanosuspensions: a promising drug delivery strategy. J. Pharm. Pharmacol., 2004; 56: 827-840.

89. Couvreur, P., Barratt, G., Fattal, E., Legrand, P., Vauthier, C. Nanocapsule technology: A review; Crit. Rev. Ther. Drug., 19; 2002; 99-134.

90. Santos, N.P.D.S. Nanoencapsulation of usnic acid: An attempt to improve antitumour activity and reduce hepatotoxicity. Eur. J. Pharm. Biopharm., 2006; 64: 154-160.

91. Legrand, P., Barratt, G., Mosqueira, V., Fessi, H., Devissaguet, J.P. Polymeric nanocapsules as drug delivery systems: a review. S. T. P. Pharm. Sci., 1999; 9: 411-418.

92. Barratt, G.M. Therapeutic applications of colloidal drug carriers; pharm. Sci. Technol. Today., 2000; 3: 163-171.

93. Leite, E.A. Cardiotoxicity reduction induced by halofantrine entrapped in nanocapsule devices. Life Sci., 2007; 80:1327-1334.

94. Memvanga, P.B., Préa, V. Formulation design and in vivo antimalarial evaluation of lipid-based drug delivery systems for oral delivery of $b$-arteether. European journal of pharmaceutics and biopharmaceutics., 2012;82:112-119.

95. Dwivedi, P. Self-nanoemulsifying drug delivery system (SNEDDS) for oral delivery of arteether: pharmacokinetics, toxicity and antimalarial activity in Mice. RSC Advances., 2015, 126 ,467475 .
96. Saini, S., Singh, G., kalara, N., Singh, C., Geeta, Tarun. Development of nanostructured liquid crystalline formulation of antimalarial agents Artemether and Lumefantrine. World Journal of Pharmaceutical Research., 2015; 9:1811-1828.

97. Lissinda, H. Formulation and evaluation of Pheroid vesicles containing mefloquine for the treatment of malaria. 2010; 122(5-6):173-8.

98. Agnihotri, J., Gajbhiye, V., Jain, N.K. Engineered cellular carrier nanoerythrosomes as potential targeting vectors for antimalarial drug. Pharmaceutics Research Laboratory; Department of Pharmaceutical Sciences. Dr. Hari Singh Gour University, Sagar (MP). India Asian J Pharm., 2010;117-120.

99. Deu, E. Ferrous iron-dependent drug delivery enables controlled and selective release of therapeutic agents in vivo., 1998; 09-01.

100. Raut, D.B., Sakhare, R.S., Dadge, K.K., Halle, P.D. Resealed erythrocyte drug delivery: a review. Int. Jour. In pharmacy and chemistry., 2013; 3(2):198-207.

101. Battigelli, A., Moyon, C.M., Bianco, A. Carbon nanomaterials as new tools for immunotherapeutic applications. Journal of Materials Chemistry B., 2014;2:6144-6156.

102. Rathee, P. On the efficacy of malaria DNA vaccination with magnetic gene vectors. J. Control. Release., 2013; 168: 10-17.

103. New-target-blocks-malaria-invasion-and-transmission https://www.genengnews.com/topics/translational-medicine/newtarget-blocks-malaria-invasion-and-transmission/. Accessed on: 15th April, 2012.

104. Antimalarial tea -- from herbal remedy to licensed phytomedicinehttp://m

https://www.sciencedirect.com/science/article/abs/pii/S0006291X060 26441oscow.scihub.bz/b46d6df. Accessed on: 12th April, 2012.

105. Normal blood cell as carriers. http://www.theguardian.com/global-development-professionalsnetwork/2013/apr/ . Accessed on: 14th April, 2012.

106. New malaria strategy proposes using unaffected red blood cells as drug carriers. http://www.ibecbarcelona.eu/new-malariastrategy-proposes-using-unaffected-red-blood-cells-as-drug-carriers/. Accessed on: 15th April 2012.

107. Ersmark, K., Samuelsson, B., Hallberg, A. Plasmepsins as potential targets for new antimalarial therapy. Med Res Rev., 2006 Sep; 26(5):626-66.

108. Treating malaria using a simple magnetic-resonance method. http://innovation.columbia.edu/technologies/m09014_treating-malaria-using-a-simple-magnetic-resonance-method.

Accessed on: 15th April, 2012.

109. Malaria breakthrough by Melbourne researchers at Walter and Eliza Hall Institute. https://www.heraldsun.com.au/news/malariabreakthrough-by-melbourne-researchers-at-walter-and-eliza-hallinstitute/news-story/5169df6aeaab6ca2e144aa998fc0880a. Accessed on: 12th April, 2012.

110. Flannery, E.L., Chatterjee, A.K., Elizabeth A. Winzeler1 Antimalarial Drug Discovery: Approaches and Progress towards New Medicines. Nat Rev Microbiol., 2013; 11(12): 849-862.

111. AguiarI, A.C.C., RochaI, E.M.D., SouzaI, N.B.D, Françal, T.C.C., Krettli, A. New approaches in antimalarial drug discovery and development - A Review. Mem Inst. Oswaldo Cruz, Rio de Janeiro, 2012:107(7): 831-845. 
Indo Global Journal of Pharmaceutical Sciences, 2021; 11(1): 15-27

112. Biot, C., Nosten, F., Fraisse, L., Minassian, D.T., Khalife,

J., Dive, D., The Antimalarial Ferroquine: from bench to clinic,

Parasite., 2011;18:207-214.

Indo Global Journal of Pharmaceutical Sciences( ISSN 2249 1023; CODEN- IGJPAI; NLM ID: 101610675) indexed and abstracted in CrossRef (DOI Enabling), CNKI, EMBASE (Elsevier), National Library of Medicine (NLM) Catalog (NCBI), ResearchGate, Publons (Clarivate Analytics), CAS (ACS), Index Copernicus, Google Scholar and many more. For further details, visit http://iglobaljournal.com 